\title{
Unveiling Metal-Cage Hybrid States in a Single Endohedral Metallofullerene
}

\author{
Kedong Wang, ${ }^{1}$ Jin Zhao, ${ }^{1}$ Shangfeng Yang, ${ }^{2}$ Lan Chen,,${ }^{1}$ Qunxiang Li,${ }^{1}$ Bing Wang, ${ }^{1}$ Shihe Yang, ${ }^{2}$ Jinlong Yang,,$*$ \\ J. G. Hou, ${ }^{1, *}$ and Qingshi Zhu ${ }^{1}$ \\ ${ }^{1}$ Structure Research Laboratory and Laboratory of Bond Selective Chemistry, University of Science and Technology of China, \\ Hefei 230026, People's Republic of China \\ ${ }^{2}$ Department of Chemistry, The Hong Kong University of Science and Technology, \\ Clear Water Bay, Kowloon, Hong Kong, People's Republic of China \\ (Received 14 July 2003; published 31 October 2003)
}

\begin{abstract}
The local structural and electronic properties of individual metallofullerenes are studied using scanning tunneling microscopy, scanning tunneling spectroscopy, and theoretical simulations. The energy-resolved metal-cage hybrid states of a single endohedral metallofullerene $\mathrm{Dy} @ \mathrm{C}_{82}$ isomer I have been spatially mapped, supporting a complex picture consisting of the orbital hybridization and charge transfer for the interaction between the cage and the metal atom. The relative position of the encapsulated Dy atom inside the cage and the molecular orientation on the surface have been inferred by comparing the experimental results with theoretical simulations. The combined technique provides promising applications in the fields of in situ characterization and diagnostics of metallofullerene-based nanodevices.
\end{abstract}

DOI: 10.1103/PhysRevLett.91.185504

PACS numbers: 61.48.+c, 31.15.Ew, 68.37.Ef, 73.22.-f

Endohedral metallofullerenes have been a subject of intensive investigation in recent years, not only because of their structural and electronic novelties but also because of their promising electronic, optical, and biomedical applications [1]. The location of metal atoms inside the fullerene cage and the metal-cage interaction are two central issues. Various diffraction, spectroscopy, and microscopy techniques have been used to characterize metallofullerenes [2-7]. Most of them require macroscopic quantities of metallofullerenes and give either ensemble averaged or spatially averaged results. It has been a challenging task to characterize the local properties of isolated metallofullerene molecules [8-11]. Here we show that scanning tunneling microscopy (STM) can be used to detect the encapsulated metal atom inside a fullerene cage. The energy-resolved metal-cage hybrid states of a single endohedral metallofullerene Dy@ $\mathrm{C}_{82}$ isomer I have been spatially mapped using scanning tunneling spectroscopy (STS). Compared with other states of the metallofullerene, these hybrid states provide unique information on the location of metal atoms inside the fullerene cage and the metal-cage interaction. The relative position of the encapsulated Dy atom inside the cage and the molecular orientation on the surface have been inferred by comparing the experiments with theoretical simulations.

The Dy@ $\mathrm{C}_{82}$ isomer I is a metallofullerene in which the Dy atom lies along a $\mathrm{C}_{2}$ axis on the six-membered ring of the $\mathrm{C}_{2 v}-\mathrm{C}_{82}$ cage [6]. Details for the preparation and separation of Dy $@ \mathrm{C}_{82}$ isomer I were described elsewhere [12,13]. A small amount of solid powder of Dy@ $\mathrm{C}_{82}$ was obtained in a crucible by evaporation of solvent in a dry box. The experiments were conducted in an ultrahigh vacuum STM chamber with a base pressure of $3 \times 10^{-11}$ Torr. We first evaporated one monolayer $\mathrm{Ag}$ onto a $\mathrm{Si}(111)$ surface to form a $(\sqrt{3} \times \sqrt{3})-\mathrm{Ag}$ surface and then a submonolayer of Dy $@ \mathrm{C}_{82}$ molecules was deposited on the Ag surface. All STM and STS measurements were performed at $5 \mathrm{~K}$ using an OMICRON cryostat GmbH STM with W tips that had been subject to careful cleaning treatments. Figure 1(a) shows a typical large scale STM image of submonolayer Dy@ $\mathrm{C}_{82}$ adsorbed on the Ag surface. Bright spots correspond to the individual Dy@ $\mathrm{C}_{82}$ molecules. Figure 1(b) is a higher resolution image showing the internal patterns of two Dy $@ \mathrm{C}_{82}$ molecules. We found that there are several kinds of internal patterns of the Dy $@ \mathrm{C}_{82}$ molecules adsorbed on the surface at the same bias voltage, indicating multiple molecular adsorption configurations. Since a normal STM topological image reflects the integrated local density of states (LDOS) of the sample from the Fermi level to the sample bias voltage $V$, a $d I / d V$ spatial map is approximately proportional to the LDOS at the energy of $e V$ $[14,15]$. Our $d I / d V$ spectra were measured by lock-in technique with a modulation signal of $15 \mathrm{mV}(\mathrm{rms})$ and $2000 \mathrm{~Hz} . d I / d V$ spatial maps were obtained by positioning the STM tip at each point at constant current and then measuring $d I / d V$. Figures 1(b) and 1(e) are the spatial maps of $d I / d V$ for four Dy $@ \mathrm{C}_{82}$ molecules taken at 2.1, $2.2,2.0$, and $2.1 \mathrm{~V}$, respectively. One can clearly see from these figures that there is an off-center bright spot in each of the hollow carbon cages though these molecules have different adsorption configurations. We will show that this local bright spot is related to a metal-cage hybrid state. The existence of metal-cage hybrid states will be a direct evidence for the complex picture consisting of the orbital hybridization and charge transfer for the interaction between the cage and metal atom. 


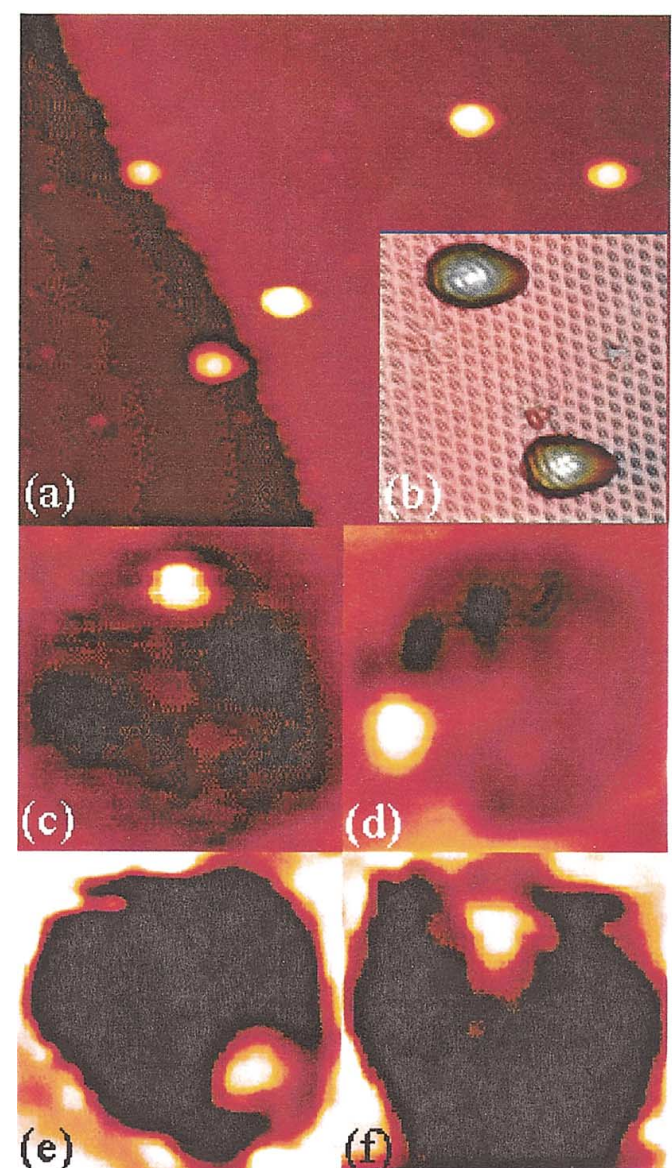

FIG. 1 (color). (a) A $500 \times 400 \AA^{2}$ STM image of submonolayer of Dy@ $\mathrm{C}_{82}$ isomer I adsorbed on $\mathrm{Si}(111)-(\sqrt{3} \times \sqrt{3})-\mathrm{Ag}$ surfaces. (b) A high resolution STM image $\left(40 \times 40 \AA^{2}\right)$ revealing the internal patterns of two Dy@ $\mathrm{C}_{82}$ molecules. (c)-(f) Scanning tunneling spectroscopy (or $d I / d V)$ images $(26.6 \times$ $26.6 \AA^{2}$ ) of individual Dy@ $\mathrm{C}_{82}$ molecules with different adsorption configurations on the Ag surface taken at 2.1, 2.2, 2.0, and $2.1 \mathrm{~V}$, respectively.

To systematically characterize the structural and electronic properties of individual Dy@ $\mathrm{C}_{82}$ molecules, we took one adsorbed Dy@ $\mathrm{C}_{82}$ molecule as an example to measure its STM images and $d I / d V$ spatial maps with different bias voltages, which are shown in Fig. 2. The internal pattern of the STM images depends strongly on the bias voltages. At the negative voltage, the molecule appears as several slightly curved bright stripes [Fig. 2(a)]. Such an intramolecular pattern has been observed in STM images of other fullerene molecules such as $\mathrm{C}_{60}$ and $\mathrm{C}_{70}$ adsorbed on different surfaces, and ascribed to the combined contributions of the fullerenes and substrates [16,17]. The large positive bias STM images [Figs. 2(b) and 2(c)] show some bright pentagon and hexagon rings. These patterns are believed to be most directly related to the cage structure of the fullerene [18]. In contrast to $C_{60}$, which has only one unique carbon site, the Dy@ $\mathrm{C}_{82}$ molecule has many nonequivalent sites

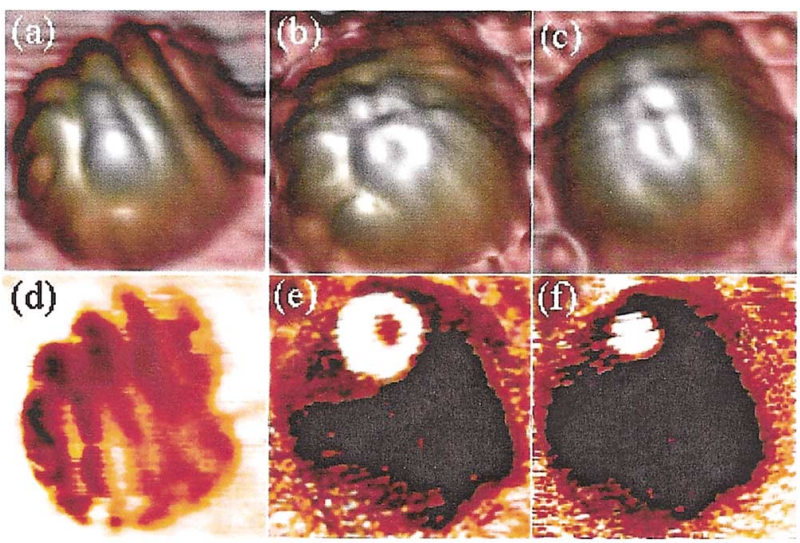

FIG. 2 (color). (a)-(c) STM images of a Dy@ $\mathrm{C}_{82}$ molecule taken at $V=-1.3,1.4$, and $1.8 \mathrm{~V}$. (d) - (f) $d I / d \mathrm{~V}$ maps of the same Dy@ $\mathrm{C}_{82}$ molecule taken at $V=-1.3,2.0$, and $2.1 \mathrm{~V}$.

due to its low symmetry. Thus, we cannot deduce the orientation of Dy@ $\mathrm{C}_{82}$ with these STM images alone as done for $\mathrm{C}_{60}$. The $d I / d V$ spatial maps of Dy@ $\mathrm{C}_{82}$ at most bias voltages we measured show either a fully dark hollow (not shown) or a bright mesh structure [Fig. 2(d)]. Only at certain positive bias voltages (2.0 and $2.1 \mathrm{~V})$, we observed a locally bright ring or dot [Figs. 2(e) and 2(f)]. These $d I / d V$ maps reveal detail in the electronic structure that cannot be observed in topographs [14].

In order to understand qualitatively the above experimental observations, we have studied theoretically the isolated Dy@ $\mathrm{C}_{82}$ molecule. Our calculations were carried out using the density functional theory with local density approximation (LDA) implemented with the $\mathrm{Dmol}^{3}$ package [19]. The Vosko-Wilk-Nusair local correlation functional [20] and all-electron calculations with scalar relativistic corrections were used. The basis set consists of the double numerical atomic orbitals augmented by polarization functions, i.e., functions with angular momentum one higher than that of the highest occupied orbital in the free atom. The Dy@ $\mathrm{C}_{82}$ molecule was constructed by placing a Dy atom into the cage of the $\mathrm{C}_{2 V}-\mathrm{C}_{82}$ isomer. In the optimized geometry, the Dy atom lies along a $\mathrm{C}_{2}$ axis on the six-membered ring of the $\mathrm{C}_{2 V}-\mathrm{C}_{82}$ cage. The distance between the Dy atom and the six nearest $\mathrm{C}$ atoms is $2.46 \AA$, in good agreement with the experimental value of $2.48 \AA$ [6]. To examine the effect of the choice of the exchange-correlation functional on the electronic structure, we performed a single-point calculation of the Dy@ $\mathrm{C}_{82}$ molecule using the generalized gradient approximation (GGA) [21]. We found that the GGA calculation gives almost the same result as the LDA calculation within a few percent.

Figure 3(a) shows the energy level diagram of a free Dy $@ \mathrm{C}_{82}$ molecule. The calculated energy gap between the highest occupied molecular orbital (HOMO) and the lowest unoccupied molecular orbital is $0.23 \mathrm{eV}$ which agrees well with the experimentally estimated value of 

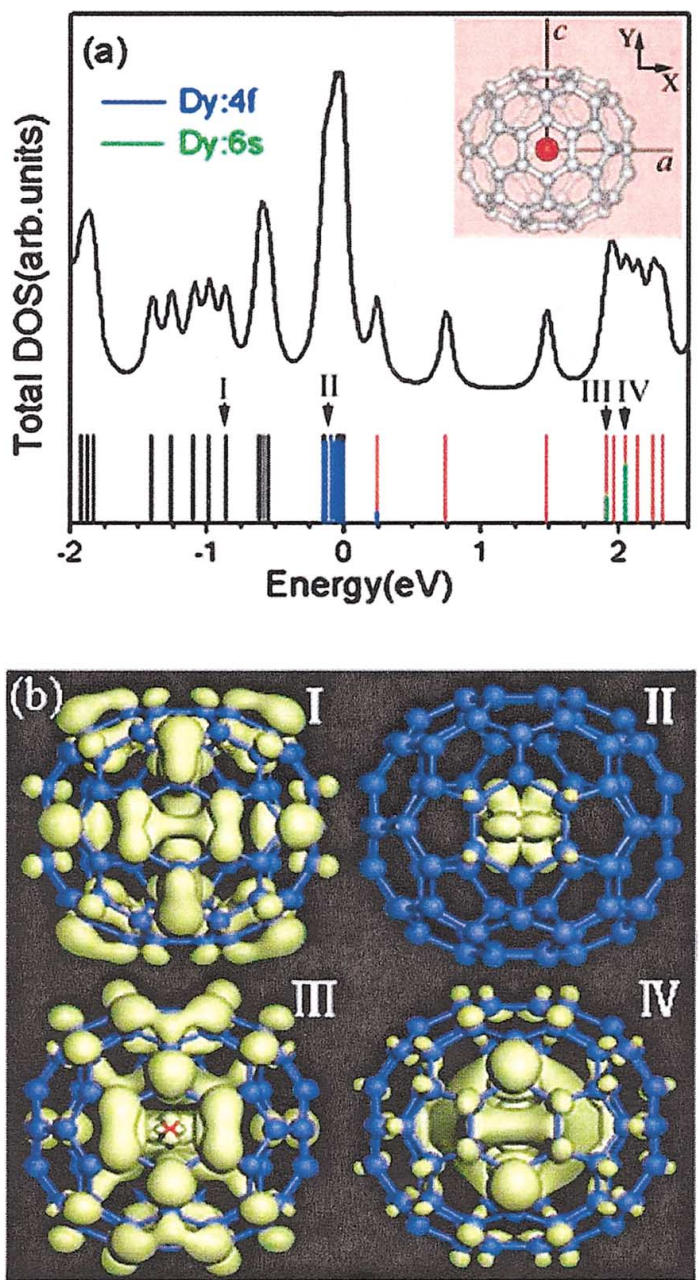

FIG. 3 (color). (a) The density of states and energy level structure of a free Dy@ $\mathrm{C}_{82}$ molecule. The zero of energy is shifted to the HOMO. The lengths and colors of lines represent orbital populations from different atomic orbitals. Inset: A standard orientation of Dy@ $\mathrm{C}_{82}$ in which the $\mathrm{C}_{2}$ axis defines the $z$ direction. (b) Isosurfaces of the electron density with isovalue of $5.0 \times 10^{-4} \mathrm{e} / \AA^{3}$ for the orbitals at $-0.9 \mathrm{eV}$ (I), $-0.12 \mathrm{eV}$ (II), $+1.95 \mathrm{eV}$ (III), and $+2.05 \mathrm{eV}$ (IV), respectively.

$0.2 \mathrm{eV}$ [7]. The orbital population analysis shows that the molecular orbitals of Dy@ $\mathrm{C}_{82}$ can be divided into three types: cage-dominated [type I, Fig. 3(b)-I], metal-dominated [type II, Fig. 3(b)-II], and metal-cage hybrid [type III, Figs. 3(b)-III and 3(b)-IV)] orbitals. In the occupied valence-band region, only types I and II orbitals exist, supporting the previously experimental result that the ionic character exclusively dominates the interaction between the encapsulated lanthanide metal atoms and the fullerene cage [6,7]. In the unoccupied conduction-band region, however, there are two metal-cage hybrid orbitals at around $2 \mathrm{eV}$ above the HOMO. In these orbitals, there is a strong hybridization between the Dy $6 s$ orbital and the $2 s$ and $2 p$ orbitals of some specific $\mathrm{C}$ atoms. This kind of hybridization can result in a very local distribution of the
LDOS on the fullerene cage. As an example, the spatial LDOS distribution of the second hybrid orbital at $2.05 \mathrm{eV}$ is presented in Fig. 3(b)-IV. The LDOS value at the two carbon atoms in the six-membered ring of the cage nearest to the Dy atom is much larger than that at other carbon atoms.

Since type I orbitals are irrelevant to the encapsulated metal atom, the LDOS of these orbitals show no direct information on the metal atom position in the fullerene cage. Type II orbitals belong to the metal atom, but they are localized inside the fullerene cage, and the LDOS of these orbitals cannot be detected by the STS. In order to detect the metal atom in the fullerene cage, mapping the metal-cage hybrid orbitals becomes the crux of the matter. According to Fig. 3(b)-IV, one can expect that the $d I / d V$ map of the hybrid orbital at $2.05 \mathrm{eV}$ should be two or one bright dot, depending on the orientation of the molecule. This explains the observed off-center bright spot in the hollow carbon cage at around $2 \mathrm{~V}$ for different adsorbed Dy@ $\mathrm{C}_{82}$ molecules. In principle, we can determine where the metal atom is in the cage and, hence, the molecular orientation on the surface by matching the simulated LDOS distribution of the hybrid orbitals and STM images with the experimental $d I / d V$ maps and STM images. Figure 4 shows the simulated STM images and $d I / d V$ maps by assuming the Dy@ $\mathrm{C}_{82}$ molecule adsorbed on the surface with an orientation $(\alpha=$ $168.9^{\circ}, \beta=96.0^{\circ}$, and $\gamma=207.0^{\circ}$ ) relative to a standard orientation [see the inset of Fig. 3(a)]. The agreement between experiment [Figs. 2(b), 2(c), 2(e), and 2(f)] and theory is quite good. We notice that to understand all observed STM images and $d I / d V$ maps, the moleculesubstrate interaction and STM tip trajectory should be taken into account.

In all of the experimental $d I / d V$ maps, the metal atom is found to be on the side of the cage, suggesting that the Dy $@ \mathrm{C}_{82}$ molecule is found on the substrate in a small subset of all of the possible orientations. This observation could be understood by noting that, instead of a perfect sphere, the $\mathrm{C}_{2 V}-\mathrm{C}_{82}$ cage is actually an ellipsoid with $a=$ $7.96 \AA, b=8.07 \AA$, and $c=7.45 \AA$ with the Dy atom being on the $b$ axis. Because the $c$ axis is shorter than the $a$ and $b$ axes, the ellipsoid prefers the adsorption configurations with the $a b$ plane approximately parallel to the surface of the substrate. As a result, only one of the two bright spots due to the $\mathrm{C}$ atoms neighboring the Dy atom can be seen. According to theoretical calculations [22,23], the existence of the metal-cage hybrid states in metallofullerenes is a universal phenomenon. Our experimental observations of the metal-cage hybrid states support that the orbital hybridization and charge transfer constitute a complete picture for the interaction between the cage and metal atom [3,13]. Detecting the energy-resolved metalcage hybrid states of a single endohedral metallofullerene provides not only the information for the metal-cage interaction, but also the geometrical and orientational 


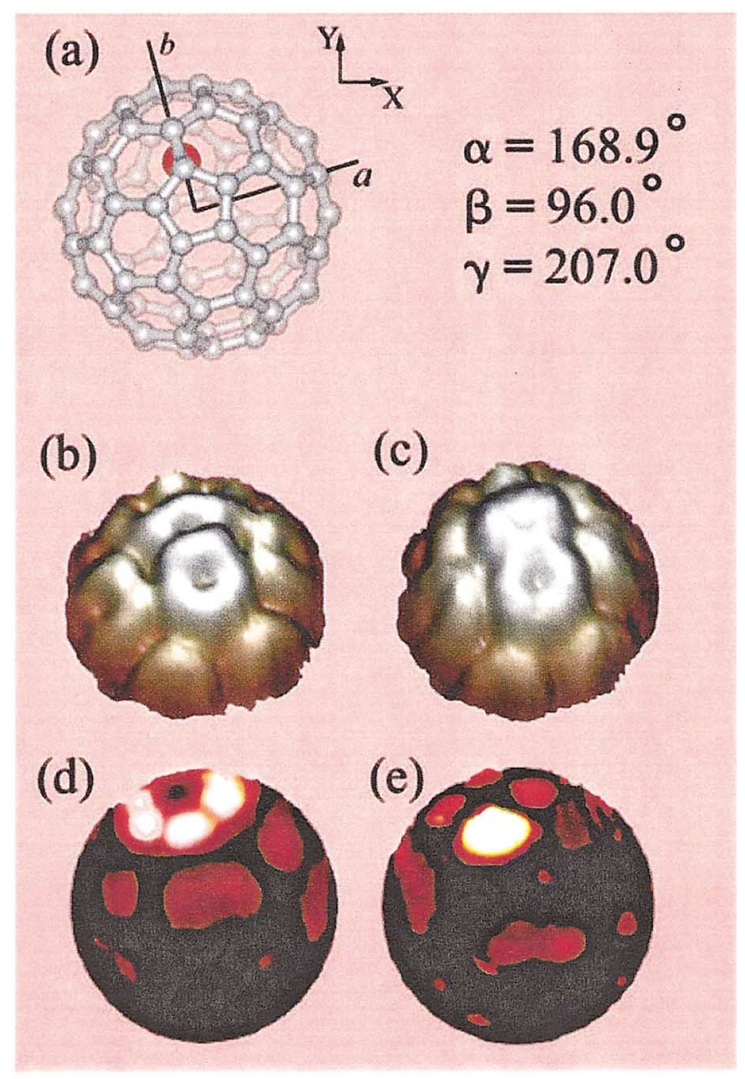

FIG. 4 (color). Simulated STM images and $d I / d V$ maps of a Dy@ $\mathrm{C}_{82}$ molecule. (a) Molecular orientation on the Ag surface. The three Euler angles are relative to the standard orientation of Dy@ $\mathrm{C}_{82}$ defined in the inset of Fig. 3(a). (b),(c) Simulated STM images for $V=1.4$ and 1.8 V. (d), (e) Simulated $d I / d V$ maps at $E=1.95$ and $2.05 \mathrm{eV}$.

information about the metallofullerene. This technique may find interesting applications in the fields of in situ characterization and diagnostics of metallofullerenebased nanodevices.

This work is partially supported by the National Project for the Development of Key Fundamental Sciences in China (G1999075305, G2001CB3095), by the National
Natural Science Foundation of China (50121202, 50132030, 20025309, 10074059), by the Foundation of Ministry of Education of China, and by the Foundation of the Chinese Academy of Science. S. Y. acknowledges the support from an RGC grant administered by the UGC of Hong Kong.

*Author to whom correspondence should be addressed. Electronic address: jghou@ustc.edu.cn \& jlyang@ustc.edu.cn

[1] H. Shinohara, Rep. Prog. Phys. 63, 843 (2000).

[2] C. H. Park et al., Chem. Phys. Lett. 213, 196 (1993).

[3] B. Kessler et al., Phys. Rev. Lett. 79, 2289 (1997).

[4] S. Hino et al., Chem. Phys. Lett. 281, 115 (1997).

[5] E. Nishibori, M. Takata, M. Sakata, M. Inakuma, and H. Shinohara, Chem. Phys. Lett. 298, 79 (1998).

[6] S. Iida et al., Chem. Phys. Lett. 338, 21 (2001).

[7] Y. Kubozono et al., Phys. Rev. B 67, 115410 (2003).

[8] M. Takata et al., Nature (London) 377, 46 (1995).

[9] K. Suenaga et al., Science 290, 2280 (2000).

[10] K. Suenaga et al., Phys. Rev. Lett. 90, 055506 (2003).

[11] A. Taninaka et al., Nano Lett. 3, 337 (2003).

[12] H. Huang and S. H. Yang, Chem. Mater. 12, 2715 (2000).

[13] H. Huang, S. H. Yang, and X. Zhang, J. Phys. Chem. B 104, 1473 (2000).

[14] X. Lu, M. Grobis, K. H. Khoo, S. G. Louie, and M. F. Crommie, Phys. Rev. Lett. 90, 096802 (2003).

[15] H. Kim et al., Phys. Rev. Lett. 90, 216107 (2003).

[16] J. G. Hou et al., Phys. Rev. Lett. 83, 3001 (1999).

[17] T. Sakurai et al., Prog. Surf. Sci. 51, 263 (1996).

[18] H. Q. Wang et al., Phys. Rev. B 63, 085417 (2001).

[19] $\mathrm{Dmol}^{3}$ is a density-function-theory-based package with atomic basis distributed by Accelrys [B. Delley, J. Chem. Phys. 92, 508 (1990)].

[20] S. H. Vosko, L. Wilk, and M. Nusair, Can. J. Phys. 58, 1200 (1980).

[21] J. P. Perdew and Y. Wang, Phys. Rev. B 45, 13244 (1992).

[22] J. Lu, X. Zhang, X. Zhao, S. Nagase, and K. Kobayashi, Chem. Phys. Lett. 332, 219 (2000).

[23] K. Kobayashi and S. Nagase, Chem. Phys. Lett. 282, 325 (1998). 